\title{
New Thermal Imager for Long Range Surveillance
}

\author{
J.Fritze, H. Schlemmer \\ Cassidian Optronics GmbH, Carl-Zeiss-Str.22, 73447 Oberkochen, Germany \\ joerg.fritze@cassidian-optronics.com
}

\begin{abstract}
The concept and design of a new thermal imager branded Attica M-ER is presented which is dedicated to long range border surveillance applications. The camera makes use of a 1280x1024 pixel CMT or InSb detector being sensitive in the MWIR atmospheric window between 3 and 5 micron. For high quality imaging with fields of view between $0.6^{\circ}$ and $30^{\circ}$ a $50 \mathrm{x} \mathrm{IR}$ optical zoom lens is designed comprising 11 lenses. Five of the lenses are aspheric and one in addition with a diffractive surface structure. Specially adapted image processing algorithms for homogenization, edge enhancement and dynamic compression as well as automatic functions (focus, contrast, brightness, etc.) are completing the package. Video clips of a first application and examples of image simulation are also presented.
\end{abstract}

Key words: Infrared, Thermal Imager, Optics, Image processing, surveillance system

\section{Boarder Surveillance Applications}

Securing the home country or the maritime borders of a country needs long range observation capabilities. To fulfill the tasks also at night, under adverse weather conditions or under the influence of strong air turbulence (e. g. in the desert states) the employment of thermal imagers is highly favorable. To enhance the situational awareness of the observer a wide field of view (FOV) is needed for a good survey as well as a narrow FOV for identifying a target of interest. This makes necessary an IR zoom lens with high zoom factor. To provide high resolution in every FOV which is the first essential of achieving a long observation range a detector array with a large number of pixels is crucial. The concept of the new Attica M-ER thermal imager was established especially to meet these objectives. The unique feature of the ATTICA M-ER being the biggest member of the ATTICA M family [1], is the use of a megapixel detector and a new powerful video electronics.

\section{Optical design and device configuration}

The zoom lens comprises 3 movable units. Besides compensator and variator a third unit was found to be necessary for compensation of the pupil aberrations caused by the very large zoom factor. All together 11 lenses are employed, among these are 5 aspheric lenses and one diffractive optical element. Most of the lenses are made from Silicon the rest is from
Germanium and Calcium fluoride. As the diameter of the front lens is limited to some $250 \mathrm{~mm}$ by reasons of manufacturability a warm shield is inserted directly in front of the detector window whenever the zoom is tuned to an extremely narrow FOV. The lens can be zoomed continuously between a wide FOV of $30^{\circ} \times 24^{\circ}$ and an extreme narrow FOV of $0.6^{\circ} \times$ $0.48^{\circ}$ resulting in a zoom factor of 50 .

In addition to the 50x optical zoom a digital continuous zoom factor of up to $8 x$ can be applied.

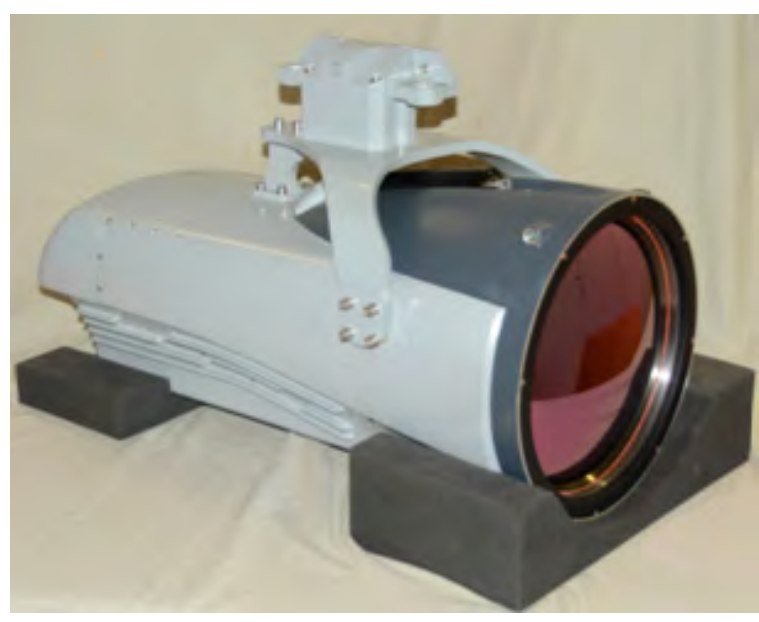

Fig. 1:ATTICA M-ER

The zoom lens is combined with a megapixel detector $(1280 \times 1024)$ CMT or InSb being sensitive in the MWIR range between $3.7 \mu \mathrm{m}$ and $5.1 \mu \mathrm{m}$. Both detector types are cooled by integral rotary cooler (RICOR, K548) with 0.75 
W cooling power. Optionally also a smaller detector with $640 \times 512$ pixels can be used in case HD video is not needed or not possible due to the system infrastructure (cables, slip rings, displays, recorders, etc.) being not suitable for high resolution video. If a $640 \times 512$ detector is used the FOVs are reduced by a factor of 2, without any influence to the ranges.

To reduce the mechanical length of the thermal imager to an acceptable length, the optical path is $U$ - folded. The overall length of the housed device is about $700 \mathrm{~mm}$, the weight is approximately $27 \mathrm{~kg}$.

A motorized cover protects the front lens in case of sand storm or even during transport.

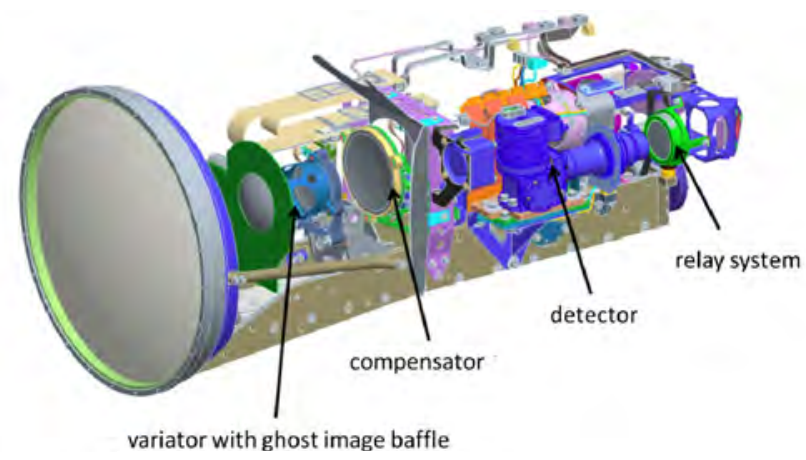

Fig. 2: Optical bench including detector/ cooler assembly

\section{Video electronics}

The video electronics is the central part of the ATTICA M. The electronic unit comprises the main PC board and an interface control board which is connected with board to board connectors to the main board.

In addition a video compression module with $\mathrm{H} 264$ compression is connectable. If connected, communication and compressed video stream is available via $1 \mathrm{Mbit}$ Ethernet interface.

The image processing electronics operates with a XILINX Virtex 6 FPGA and a DaVinci digital signal processor with $900 \mathrm{MHz}$ clock rate.

The FPGA can operate two independent video paths with different graphics and geometrical and dynamical resolution. It is i.e. possible to put a HD-SDI video stream with graphics overlay on a monitor and in parallel provide a 15 bit dynamic video to a tracker.

The video electronics provides a lot of different video output standards (many of them in parallel):
- analog: CCIR, 720p25/p30/p50/p60, 1080p25/p30/i50/i60

- digital uncompressed: 720p25/p30/p50/ p60, 1080p25/p30/i50/i60

- digital compressed: $1280 \times 72025$ or 30 fps, $1920 \times 1080,25$ or $30 \mathrm{fps}$

Simultaneously Ethernet, RS422 and CANopen are available for communication.

Beneath communication and image processing, all functions of the objective lens are realized by video electronics.

\section{Image processing}

The quality of the images displayed is further improved by a variety of software algorithms which are optimized for the Attica M-ER thermal imager.

A very important criterion on thermal images is a homogeneous image impression. Homogeneity is affected by defect or blinking pixels, fixed pattern noise; caused by dispersion and non-linearity of the responsivity of individual detector pixels as well as decrease of focal plane array sensitivity towards FOV edge caused be $\cos ^{4}$ law. Furthermore, vignetting, stray light and ghost images within the thermal imager could degrade image homogeneity.

An effective homogenization strategy is necessary to get a sufficient image impression under any environmental conditions. On ATTICA M-ER several gain correction tables for the FOVs with and without warm shield activated $a$ are measured and stored during assembly. An offset correction including defect pixel and blinker search is performed after each cool down and in addition can be commanded if needed. Since individual pixel offsets depend on scene temperature, we found that a proper method for offset correction is to use a totally defocussed image from the scene itself. In this case it is assured, that correction is done at an averaged scene temperature. An online scene based blinker search detects and after a short time removes randomly appearing defects out of the image by replacing pixel information with information from adjacent non defect pixels.

Another field of image processing is to provide the user a maximum of dynamics in the image. Under normal light conditions (not too bright, no reflexes on screen) an untrained human eye can differentiate between 100 and 250 grey levels. The detector itself provides a signal with 16 bit dynamic range (equivalent 65500 grey levels) which are compressed by contrast and brightness settings to 10 Bit (equivalent 1024 grey levels) in the video output. Beneath 
contrast and brightness adjustment ATTICA MER provides histogram equalization as well as a local adaptive dynamic compression (LADC) filter which evaluates locally (16x16 pixel) on 14 Bit base signal which grey levels has to be
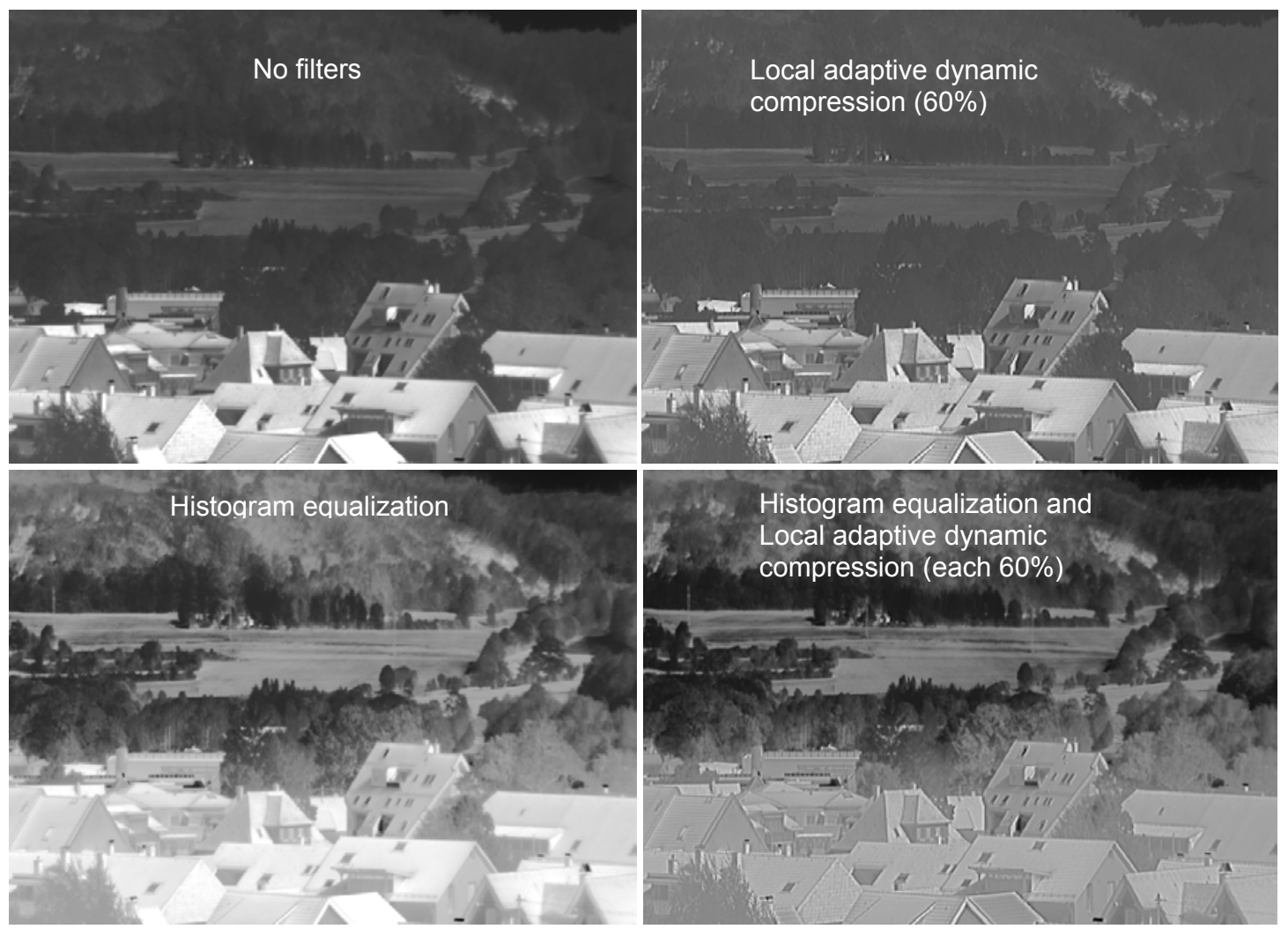

Fig. 3: Effects of mixing histogram equalization and Local adaptive dynamic compression filters with unfiltered Image.

The human eye by far can resolve more color differences than grey levels (e.g. $0,1 \mathrm{~nm}$ within the green wavelength) Therefore a false color display is foreseen as an option. In another functional mode a grey level band can be specified in between objects are displayed in red color. This makes it more easy for the observer to spot interesting objects (which are warm typically) on the screen.

For detecting distant objects noise reduction in the image is essential. To achieve this, the average well-fill of the detector pixels can be influenced by adjusting integration time. The maximum is given approximately by the inverse of the frame rate, e.g. $50 \mathrm{~Hz}$ results in $<20 \mathrm{~ms}$. For the video outputs according to standards i50 (i60) it is possible to reduce the image capture frequency of the detector from $50 \mathrm{~Hz}(60 \mathrm{~Hz})$ to $25 \mathrm{~Hz}(30 \mathrm{~Hz})$, in order to enlarge the maximum available integration time by factor of two.

An appropriate means to reduce the temporal noise (NETD) is image stacking (averaging of displayed. In addition the user is enabled to mix continuously histogram equalization and LADC. The effect of mixing filters is shown in figure 3. 
availability is mainly influenced by the Meantime to repair (MTTR) of a component which failed. The MTTR is the time between breakdown of component and restart of the full functionality. In many cases shipment to factory, repair and reimport possibly into a foreign country would be too time-consuming. Thus it is highly favorable to install a great part of the service near the customer with the support of local partners. Stock holding of complete thermal imagers for exchange in case of failure would increase the cost immensely. To avoid high stock cost, our service concept foresees the exchange of components with high failure rate in field. On thermal imager the components with high failure rates are typically detector/ cooler unit, and electronics. The mechanical design provides accessibility of all those components by opening covers. A special mechanical interface on the detector/ cooler unit enables exchange without readjustment of line of sight, only 2 point correction is necessary, which can be done easily by means of a blackbody. Specific parameters, necessary to drive the detector and electronics properly as well as SW updates can be downloaded easily via a webserver interface.

ATTICA M-ER contains a data logger, which during lifetime of thermal imager counts and stores number of cool down cycles, operating hours of cooler and other components, temperature profile which the devices was faced to. In addition occurred failures and warnings are recorded, which together with a build in test system helps to identify failed components.

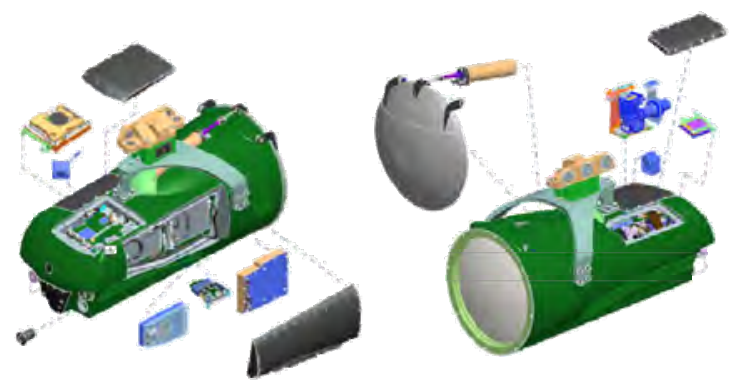

Fig. 4:. Spare part concept of ATTICA M-ER

\section{First measurement results}

Measurements of NETD (Noise Equivalent Temperature Difference) and IETD (Inhomogeneous Effective temperature Difference) as well as MTDP (Minimum Temperature Difference Perceived) were measured on one of the first available units (see table 1).

Tab. 1: Measured Noise values

\begin{tabular}{|c|c|c|}
\hline & XNFOV & NFOV \\
\hline NETD & $24,6 \mathrm{mK}$ & $18,9 \mathrm{mK}$ \\
\hline IETD & $9,5 \mathrm{mK}$ & $7,2 \mathrm{mK}$ \\
\hline
\end{tabular}

Fig. 5 compares the measured MTDP in XNFOV with the theoretical values, calculated with TRM4 [2]. The measured values show a very good compliance to theoretical MTDP curves.

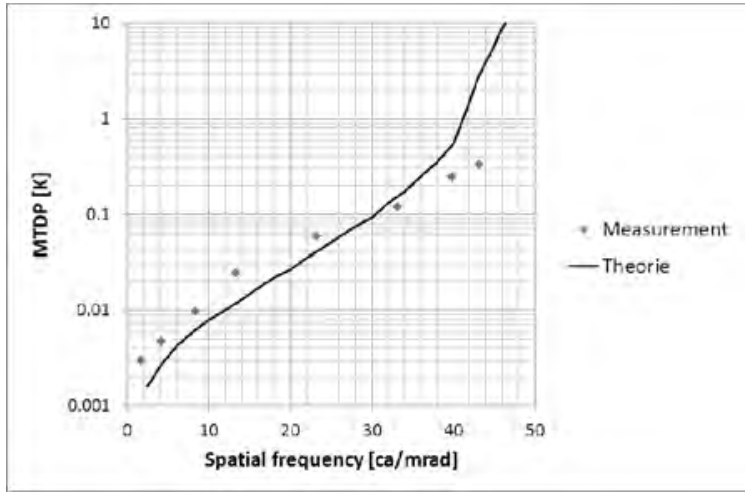

Fig. 5:. Measured and theoretical MTDP for XNFOV

Measured MTDP values are entered into the range model TRM4 in order to calculate the corresponding ranges. A NATO standard target was assumed $(2,3 \mathrm{~m} \times 2,3 \mathrm{~m}, \mathrm{dT}=2 \mathrm{~K})$. Ranges are calculated for good and worse weather conditions acc. STANAG 4347 (extinction coefficient $\sigma=0,2 / \mathrm{km}$ and $\sigma=1 / \mathrm{km}$, respectively). Probability of task fulfillment was $50 \%$ acc. to Johnson with 1 cy/target for detection, 3 cy/target for recognition and 6 cy/target for identification. Table 2 shows calculated ranges. 
Tab. 2: Calculated Range for NATO Std Target in XNFOV

\begin{tabular}{|c|c|c|}
\hline & $\sigma=0,2 / \mathrm{km}$ & $\sigma=1,0 / \mathrm{km}$ \\
\hline Detection & $24,4 \mathrm{~km}$ & $6,3 \mathrm{~km}$ \\
\hline Recognition & $17,7 \mathrm{~km}$ & $5,5 \mathrm{~km}$ \\
\hline Identification & $13,3 \mathrm{~km}$ & $4,6 \mathrm{~km}$ \\
\hline
\end{tabular}

\section{Night Owl System}

ATTICA M-ER is part of the Night Owl System which is shown in Figure 6. Beneath Thermal Imager, Night owl includes in a common housing a high resolution color camera and a laser range finder. Both main components can be directed by a pan tilt head. Communication as well as videos are send by $100 \mathrm{Mbit} / \mathrm{s}$ LAN lines. Display of the high resolution images as well as the control of the sensors is done by a touch screen monitor. Optional a separate PC provides algorithms for tracking, turbulence correction and image stabilization.

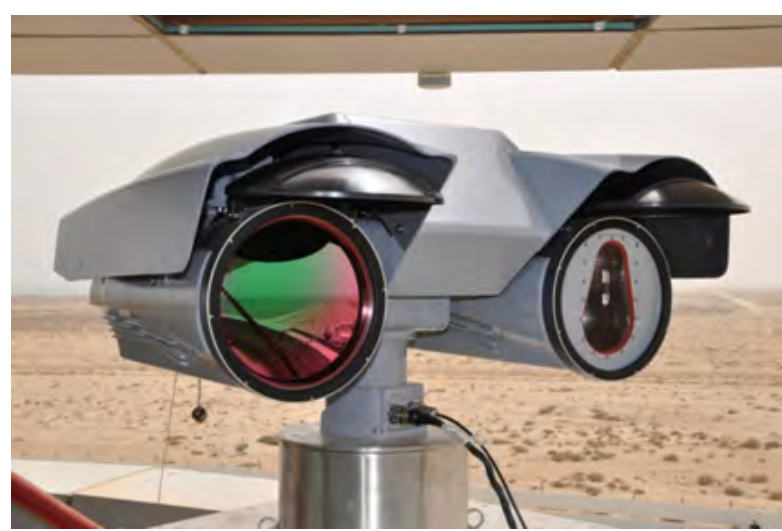

Fig. 6:.ATTICA M-ER integrated in Night Owl System

\section{References}

[1] J. Fritze, M. Münzberg, The new megapixel thermal imager family, Proc. SPIE Vol. 8012, Orlando 2011

[2] IOSB-Fraunhofer Institute für Optronik, Range Performance Model TRM4, August 2010 